\title{
INFLUÊNCIA DA IDADE E DA POSIÇÃO RADIAL NA FLEX̃̃O ESTÁTICA DA MADEIRA DE Eucalyptus grandis Hill ex. Maiden ${ }^{1}$
}

\author{
José de Castro Silva² ${ }^{2}$ Jorge Luis Monteiro de Matos $^{3}$, José Tarcísio da Silva Oliveira ${ }^{4}$ e Wescley Viana
} Evangelista ${ }^{5}$

\begin{abstract}
RESUMO - Este trabalho objetivou testar a variação da flexão estática da madeira de Eucalyptus grandis, ao longo da seção radial e sob quatro diferentes idades (10, 14, 20 e 25 anos), provenientes de talhões comerciais. As amostras foram retiradas da prancha diametral, de cada uma das 16 árvores (quatro para cada idade), tomadas de quatro posições eqüidistantes $(0,33,66$ e $100 \%)$, no sentido medula-casca, com oito repetições por posição. Verificou-se que os módulos de elasticidade (MOE) e de ruptura (MOR) apresentaram valores médios de 129.230 $\mathrm{kgf} / \mathrm{cm}^{2}$ e $854 \mathrm{kgf} / \mathrm{cm}^{2}$, respectivamente, e ambos se mostraram positivamente correlacionados com a idade e com a posição radial, no sentido medula-casca. Os maiores valores foram conseguidos nas madeiras de 20 anos de idade e localizadas na região mais próxima da casca.
\end{abstract}

Palavras-chave: Eucalyptus grandis, flexão estática, idade e variação radial.

\section{INFLUENCE OF THE AGE AND OF THE RADIAL POSITION IN STATIC OF THE WOOD OF Eucalyptus grandis Hill. ex. Maiden}

\begin{abstract}
The objective of this work was to study the static bending variation of Eucalyptus grandis wood along the radial plane at four different ages (10-14 20 and 25 years), from commercial stands. The samples were removed from a diametrical board on each of sixteen trees (four from each age); taken from four equidistant positions $(0,33,66$ and 100\%), in the pith-to-bark direction, with six replicates per position. It was found that the elasticity modulus (MOE) showed mean values of $129.230 \mathrm{kgf} / \mathrm{cm}^{2}$, and the rupture modulus (MOR) showed mean values of $854 \mathrm{kgf} / \mathrm{cm}^{2}$, both showing to be positively correlated with age and radial position in the pith-to-bark direction. The greatest values were obtained from twenty-year-old wood and located near the bark.
\end{abstract}

Keywords: Eucalyptus grandis, static bending, age and radial variation.

\section{INTRODUÇÃO}

Segundo Moreira (1999), a fibra é considerada a fonte de elasticidade e resistência da madeira. Como material anisotrópico, a madeira possui propriedades mecânicas únicas e independentes nas direções dos três eixos ortogonais. As suas propriedades, portanto, variam com a direção da carga em relação aos seus três eixos. O módulo de ruptura (MOR) e o módulo de elasticidade (MOE) são dois parâmetros normalmente determinados nos testes de flexão estática e são de grande importância na caracterização tecnológica da madeira; ambos dão uma boa aproximação da resistência do material, constituindo-se, na prática, parâmetros de grande aplicação na classificação dos materiais.

\footnotetext{
${ }^{1}$ Recebido em 05.10.2004 e aceito para publicação em 10.08.2005.

${ }^{2}$ Departamento de Engenharia Florestal da UFV - 36570-000 Viçosa-MG.

${ }^{3}$ DETR/UFPR, Av. Lothário Meissner, 3400, Jardim Botânico 80210-120 Curitiba-PR.

${ }^{4}$ Departamento de Engenharia Rural, UFES, Caixa Postal 16, 29500-000 Alegre-ES.

${ }^{5}$ Programa de Pós-Graduação em Engenharia Florestal / UFV, 36571-000 Viçosa-MG.
} 
Segundo Tsoumis (1991), a massa específica, a retratibilidade e a resistência aos esforços de flexão estática (MOE e MOR) são considerados os mais importantes para a madeira sólida, dentre os parâmetros físicomecânicos utilizados. Os seus valores expressam a combinação de diversos fatores, incluindo a constituição morfológica, anatômica e química da madeira. Segundo Forest... (1974), Kollmann e Côté (1968), as propriedades mecânicas da madeira são fortemente influenciadas por fatores diversos, como idade da árvore, ângulo da grã, teor de umidade, temperatura, constituintes químicos, fadiga, apodrecimento, massa específica, constituição anatômica, duração da tensão e da deformação, radiação nuclear, falhas na madeira, presença de nós e outros defeitos. Moreira (1999) reiterou que as propriedades mecânicas apresentam uma associação significativa com muitas das características anatômicas, em especial aquelas que dizem respeito às fibras e vasos. Segundo Klock (2000), embora o módulo de elasticidade não ofereça informações completas e reais sobre o comportamento de determinado material, pode-se concluir que valores altos de MOE indicam alta resistência e baixa capacidade de deformação do material, qualificandoo para fins estruturais.

Lima et al. (2000), estudando vários clones de Eucalyptus grandis, verificaram que o módulo de ruptura (MOR) e o módulo de elasticidade (MOE) aumentaram seus valores no sentido medula-casca. Esses autores salientaram que, embora ambos estivessem relacionados com a massa específica, fatores supostamente não importantes para a massa específica, como ângulo da grã, ângulo da microfibrila e defeitos microscopicamente imperceptíveis, passam a sê-lo nos módulos de ruptura e de elasticidade. Este trabalho teve como objetivo avaliar os módulos de elasticidade (MOE) e ruptura (MOR) da madeira de Eucalyptus grandis de quatro diferentes idades (10, 14, 20 e 25 anos) e quatro diferentes posições radiais $(0,33$, 66 e $100 \%)$, no sentido medulacasca.

\section{MATERIAL E MÉTODOS}

O material utilizado neste estudo foi obtido de plantios comerciais de Eucalyptus grandis Hill ex. Maiden, com idades de 10, 14, 20 e 25 anos, procedentes da Fazenda Monte Alegre, da KLABIN Papel e Celulose S. A., localizada no município de Telêmaco Borba, no Estado do Paraná. O esquema de retirada das amostras está ilustrado na Figura 1.

R. Árvore, Viçosa-MG, v.29, n.5, p.795-799, 2005

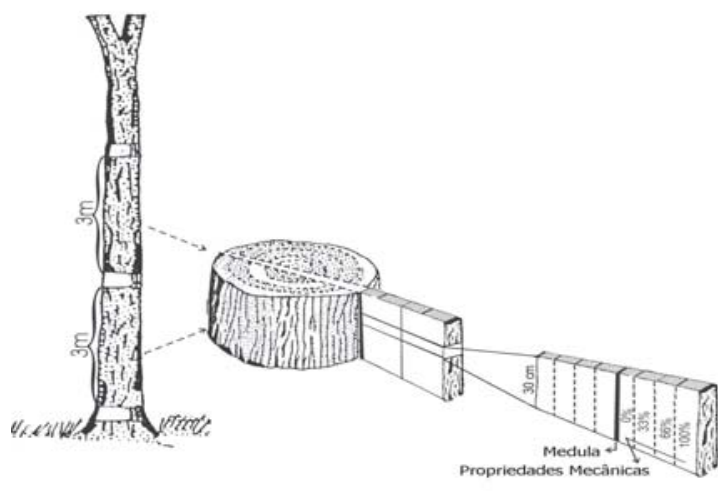

Figura 1 - Retirada das amostras para ensaios de propriedades mecânicas.

Figure 1 - Layout of mechanical properties sample location within the tree trunk.

Utilizaram-se as duas primeiras toras, de dimensões comerciais, de cada uma das 16 árvores (quatro para cada idade), quando se tomaram amostras de quatro posições eqüidistantes da prancha diametral $(0,33$, 66 e $100 \%$ ) no sentido medula-casca, com oito repetições por posição. Retiraram-se oito corpos de prova da parte mediana da prancha, com dimensões nominais de 2 x 2 x $30 \mathrm{~cm}$, sendo a última medida na direção longitudinal. Após a confecção dos corpos-de-prova, as peças foram acondicionadas na câmara climática, até atingirem peso constante, a um teor de umidade de $12 \%$. Os testes de flexão estática obedeceram às normas da ASTM (1995), com algumas adaptações, e foram realizados numa máquina universal de ensaios EMIC, gerenciada por um microcomputador. O delineamento experimental foi o inteiramente casualizado, procedendo-se à análise estatística, através da análise de variância e do teste de médias (Tukey), considerando-se os efeitos da variação da idade e da posição radial no sentido medulacasca, bem como a interação entre os efeitos, idade $\mathrm{x}$ posição radial no sentido medula-casca.

\section{RESULTADOS E DISCUSSÃO}

\subsection{Módulo de elasticidade (MOE)}

Os dados apresentados no Quadro 1 evidenciaram um aumento nos valores médios do módulo de elasticidade (MOE), em razão da idade e da direção radial, no sentido medula-casca. O valor médio para o módulo de elasticidade encontrado foi de 129.230 $\mathrm{kgf} / \mathrm{cm}^{2}$, sendo os limites individuais superior e inferior, respectivamente, de $192.668 \mathrm{kgf} / \mathrm{cm}^{2}$ (idade de 20 anos, 
na posição mais próxima da casca) e $81.438 \mathrm{kgf} / \mathrm{cm}^{2}$ (idade de 10 anos na posição mais próxima da medula). Os valores encontrados no módulo de elasticidade (MOE) estão em conformidade com os normalmente encontrados na literatura (LIMA et al., 2000; SILVA, 2002) e estão apresentados no Quadro 1.

Estatisticamente, observaram-se diferenças significativas entre os valores médios de todas as posições; situação semelhante foi verificada em todas as idades. O menor valor de módulo de elasticidade (MOE) foi encontrado na madeira de 10 anos. Os dados citados pela análise de variância confirmaram que a idade teve mais influência no módulo de elasticidade do que a posição radial no sentido medula-casca; a influência da interação desses efeitos também foi verificada, embora em menor intensidade, conforme os dados do Quadro 2.

\subsubsection{Módulo de ruptura (MOR)}

Os dados apresentados no Quadro 3 evidenciam um aumento nos valores do módulo de ruptura (MOR) em função da idade e, principalmente, da direção radial, no sentido medula-casca. Os valores encontrados no módulo de ruptura (MOR) estão em conformidade com os normalmente encontrados na literatura (LIMA et al., 2000; SILVA, 2002). Os valores médios de módulo de ruptura (MOR) estão apresentados no Quadro 3.

Verificou-se que as madeiras de 14, 20 e 25 anos, embora diferentes estatisticamente entre si, apresentaram valores muito próximos entre si no módulo de ruptura, no sentido medula-casca. Estatisticamente, observaramse diferenças significativas entre os valores médios de todas as posições; situação semelhante foi verificada em todas as idades. Os dados apresentados pela análise de variância revelam que o efeito da posição no sentido medula-casca é muito mais pronunciado do que a idade no módulo de ruptura (MOR); a influência da interação desses efeitos também foi verificada, embora em menor intensidade, conforme mostrado no Quadro 4.

Quadro 1 - Valores médios do módulo de elasticidade (MOE) em flexão estática $\left(\mathrm{kgf} / \mathrm{cm}^{2}\right)$ da madeira de Eucalyptus grandis de diferentes idades e posições, no sentido medula-casca

Table 1 -Mean values for elasticity modulus (MOE) of Eucalyptus grandis wood of different ages and at different distances in the pith-to-bark direction

\begin{tabular}{|c|c|c|c|c|c|}
\hline Tratamento & Amostras & Médias & Tukey & $\mathrm{CV}(\%)$ & $\mathrm{DP}\left(\mathrm{kgf} / \mathrm{cm}^{2}\right)$ \\
\hline Idade 10 anos & 128 & 101.313 & $a^{(1)}$ & 23,87 & 24140 \\
\hline Idade 25 anos & 128 & 130.559 & $\mathrm{~b}$ & 25,12 & 32741 \\
\hline Idade 14 anos & 128 & 135.926 & $\mathrm{c}$ & 17,76 & 24200 \\
\hline Idade 20 anos & 128 & 149.120 & $\mathrm{~d}$ & 15,39 & 22944 \\
\hline Posição 0\% (M-C) & 128 & 108.942 & a & 25,45 & 27775 \\
\hline Posição 33\% (M-C) & 128 & 118.989 & $\mathrm{~b}$ & 21,83 & 25976 \\
\hline Posição $66 \%$ (M-C) & 128 & 137.741 & $\mathrm{c}$ & 18,78 & 25840 \\
\hline Posição $100 \%$ (M-C) & 128 & 151.274 & $\mathrm{~d}$ & 16,85 & 25568 \\
\hline \multicolumn{6}{|l|}{ Interação idade X posição } \\
\hline 10 anos $x$ posição $0 \%$ & 32 & 90.313 & $\mathrm{a}$ & 28,28 & 25535 \\
\hline 10 anos $x$ posição $33 \%$ & 32 & 92.199 & $\mathrm{a}$ & 25,73 & 23707 \\
\hline 10 anos $x$ posição $66 \%$ & 32 & 107.771 & $a b$ & 22,00 & 23707 \\
\hline 14 anos $x$ posição $0 \%$ & 32 & 111.340 & abc & 21,89 & 24358 \\
\hline 25 anos $x$ posição $0 \%$ & 32 & 113.518 & abcde & 30,03 & 34122 \\
\hline 10 anos x posição $100 \%$ & 32 & 114.969 & bcde & 20,59 & 23707 \\
\hline 14 anos x posição $33 \%$ & 32 & 117.636 & bcde & 28,00 & 32962 \\
\hline 20 anos $\times$ posição $0 \%$ & 32 & 120.195 & bcde & 19,62 & 23707 \\
\hline 25 anos $x$ posição $33 \%$ & 32 & 128.845 & cdef & 17,81 & 22961 \\
\hline 20 anos x posição $33 \%$ & 32 & 137.274 & def & 16,68 & 22932 \\
\hline 14 anos x posição $66 \%$ & 32 & 138.812 & defg & 22,96 & 31915 \\
\hline 25 anos $x$ posição $66 \%$ & 32 & 150.084 & fg & 16,06 & 24126 \\
\hline 14 anos $x$ posição $100 \%$ & 32 & 152.270 & fg & 20,93 & 31915 \\
\hline 25 anos x posição $100 \%$ & 32 & 153.034 & $\mathrm{~g}$ & 14,99 & 22932 \\
\hline 20 anos $x$ posição $66 \%$ & 32 & 154.189 & $\mathrm{~g}$ & 14,70 & 22570 \\
\hline 20 anos $x$ posição $100 \%$ & 32 & 184.823 & $\mathrm{~h}$ & 14,76 & 22570 \\
\hline MÉDIA GERAL & 512 & 129.230 & & & \\
\hline
\end{tabular}

(1) Médias seguidas da mesma letra não diferem, estatisticamente, pelo teste de Tukey com significância de 5\% (p<0,05). 
Quadro 2 - Análise de variância do módulo de elasticidade (MOE) da madeira de Eucalyptus grandis de diferentes idades e diferentes posições na direção medula-casca

Table 2 - Analysis of variance of elasticity modulus (MOE) of Eucalyptus grandis wood of different ages and at different distances in the pith-to-bark direction

\begin{tabular}{lcccrr}
\hline Causa da Variação & G. L. & Soma dos Quadrados & Quadrado Médio & F & P \\
\hline PRINCIPAIS EFEITOS & 3 & & & & \\
A: Idade & 3 & $1,4093 \mathrm{E} 11$ & $4,69766 \mathrm{E} 10$ & $92,20^{*}$ & 0,0000 \\
B: Medula-casca & 9 & $1,00099 \mathrm{E} 11$ & $3,33663 \mathrm{E} 10$ & $65,49 *$ & 0,0000 \\
INTERAÇÕES & 394 & $1,54219 \mathrm{E} 10$ & $1,71354 \mathrm{E} 9$ & $3,39 *$ & 0,0006 \\
AB & 409 & $4,00736 \mathrm{E} 11$ & $5,09482 \mathrm{E} 8$ & & \\
Resíduo & TOTAL (Corrigido) & $4,7237 \mathrm{E} 11$ & & \\
\hline
\end{tabular}

* Significativo a $95 \%$ de probabilidade.

Quadro 3 - Valores médios de módulo de ruptura (MOR) em flexão estática $\left(\mathrm{kgf} / \mathrm{cm}^{2}\right)$ da madeira de Eucalyptus grandis, de diferentes idades (anos) e posições, no sentido medula-casca

Table 3 - Average values of modulus of rupture (MOR) of Eucalyptus grandis wood of different ages and at different distances in the pith-to-bark direction

\begin{tabular}{|c|c|c|c|c|c|}
\hline Tratamento & Amostras & Médias & Tukey & $\mathrm{CV}(\%)$ & $\mathrm{DP}\left(\mathrm{kgf} / \mathrm{cm}^{2}\right)$ \\
\hline Idade 10 anos & 128 & 707 & $\mathrm{a}^{(1)}$ & 56,00 & 393 \\
\hline Idade 14 anos & 128 & 859 & b & 26,36 & 393 \\
\hline Idade 25 anos & 128 & 869 & $\mathrm{c}$ & 45,59 & 385 \\
\hline Idade 20 anos & 128 & 979 & $\mathrm{~d}$ & 39,25 & 226 \\
\hline$\overline{\text { Posição 0\% (M-C) }}$ & 128 & 654 & $\bar{a}$ & 27,71 & 177 \\
\hline Posição $33 \%$ (M-C) & 128 & 767 & b & 23,53 & 179 \\
\hline Posição 66\% (M-C) & 128 & 950 & $\mathrm{c}$ & 19,00 & 179 \\
\hline Posição $100 \%$ (M-C) & 128 & 1042 & $\mathrm{~d}$ & 18,44 & 192 \\
\hline \multicolumn{6}{|l|}{ Interação idade X posição } \\
\hline 10 anos $\mathrm{x}$ posição $0 \%$ & 32 & 610 & $\mathrm{a}$ & 28,73 & 176 \\
\hline 10 anos x posição $33 \%$ & 32 & 628 & $\mathrm{ab}$ & 34,05 & 164 \\
\hline 14 anos $x$ posição $0 \%$ & 32 & 632 & $a b$ & 28,62 & 184 \\
\hline 25 anos $x$ posição $0 \%$ & 32 & 646 & $\mathrm{abc}$ & 35,92 & 236 \\
\hline 20 anos $x$ posição $0 \%$ & 32 & 725 & abcd & 22,62 & 164 \\
\hline 10 anos $x$ posição $66 \%$ & 32 & 765 & bcde & 21,43 & 164 \\
\hline 14 anos $x$ posição $33 \%$ & 32 & 770 & bcde & 29,35 & 228 \\
\hline 25 anos $x$ posição $33 \%$ & 32 & 801 & cde & 19,74 & 158 \\
\hline 10 anos $x$ posição $100 \%$ & 32 & 825 & def & 19,79 & 164 \\
\hline 20 anos $x$ posição $66 \%$ & 32 & 876 & efg & 18,10 & 158 \\
\hline 14 anos $x$ posição $66 \%$ & 32 & 981 & fgh & 22,51 & 221 \\
\hline 25 anos $x$ posição $66 \%$ & 32 & 1.018 & $h$ & 15,55 & 158 \\
\hline 25 anos x posição $100 \%$ & 32 & 1.023 & $\mathrm{~h}$ & 16,00 & 167 \\
\hline 20 anos $x$ posição $66 \%$ & 32 & 1.030 & $\mathrm{~h}$ & 14,82 & 156 \\
\hline 14 anos x posição $100 \%$ & 32 & 1.038 & $\mathrm{~h}$ & 21,27 & 221 \\
\hline 20 anos $x$ posição $100 \%$ & 32 & 1.286 & $\mathrm{i}$ & 11,87 & 156 \\
\hline Média Geral & 512 & 854 & & & \\
\hline
\end{tabular}

(1) Médias seguidas da mesma letra não diferem, estatisticamente, pelo teste de Tukey com significância de 5\% $(\mathrm{p}<0,05)$.

Quadro 4 - Análise de variância do módulo de ruptura (MOR) da madeira de Eucalyptus grandis de diferentes idades e diferentes posições na direção medula-casca

Table 4 -Analysis of variance of rupture modulus (MOR) of Eucalyptus grandis wood of different ages and at different distances in the pith-to-bark direction

\begin{tabular}{lccrcr}
\hline Causa da Variação & G. L. & Soma dos Quadrados & Quadrado Médio & F & \\
\hline PRINCIPAIS EFEITOS & & & $1,45901 \mathrm{E} 6$ & $59,70 *$ \\
A: Idade & 3 & $4,37704 \mathrm{E} 6$ & 0,0000 \\
B: Medula-casca & 3 & $8,48386 \mathrm{E} 6$ & $2,82795 \mathrm{E} 6$ & $115,71 *$ \\
INTERAÇÕES & 9 & $1,24981 \mathrm{E} 6$ & 138867,0 & 5,0000 \\
AB & 394 & $9,6291 \mathrm{E} 6$ & 24439,4 & 0,0000 \\
Resíduo & 409 & $2,46283 \mathrm{E} 7$ & & \\
TOTAL (Corrigido) & & & & \\
\hline
\end{tabular}

* Significativo a $95 \%$ de probabilidade.

R. Árvore, Viçosa-MG, v.29, n.5, p.795-799, 2005 


\section{CONCLUSÕES}

Com base nos resultados, pode-se concluir que:

a) O módulo de elasticidade (MOE) e o módulo de ruptura (MOR) foram influenciados pela idade e pela posição medula-casca das árvores.

b) A idade teve mais influência que a posição radial, no sentido medula-casca, nos valores do módulo de elasticidade, mas situação inversa foi observada no módulo de ruptura.

c) Os módulos de elasticidade e de ruptura apresentaram uma mesma tendência de crescimento em relação à idade e à posição radial, no sentido medulacasca.

d) Os maiores valores de módulo de elasticidade e de ruptura foram observados nas madeiras de idade mais elevada, à exceção da madeira de 25 anos, que apresentou valores mais reduzidos que a de 20 anos.

\section{REFERÊNCIAS BIBLIOGRÁFICAS}

AMERICAN SOCIETY FOR TESTING AND MATERIALS - ASTM - Annual book of ASTM standards - Standard Methods for Conducting Machining Tests of Wood and Wood Base Materials. ASTM D 143-93. Philadelphia: 1995. v.4., t. 10, p. 226-245.

FOREST PRODUCTS LABORATORY. Wood handbook: wood as engineering material. Washington: U.S. Department of Agriculture, 1974.521p. (Agriculture Handbook, 72).
KLOCK, U. Qualidade da madeira juvenil de Pinus maximinoi H. E. Moore. 2000. 275 f. Tese (Doutorado em Ciências Florestais) - Universidade Federal do Paraná, Curitiba, 2000.

KOllmann, F. F. P.; COTÊ, W. A. Principles of wood science and technology. Berlim: Springer-Verlag, 1968. v. 1. 592p.

LIMA, J. T.; BREESE, M. C.; CAHALAN, C. M. Variation in wood density and mechanical properties in Eucalyptus clones. In: THE FUTURE OF EUCALYPTS FOR WOOD PRODUCTS, 2000, Launceston. Procceedings... Launceston: IUFRO, 2000. p. 282-291.

MOREIRA, W. S. Relações entre propriedades físico-mecânicas e características anatômicas e químicas da madeira. 1999. 107f. Tese (Doutorado em Ciências Florestais) - Universidade Federal de Viçosa, Viçosa, 1999.

SILVA, J. C. Caracterização da madeira de Eucalyptus grandis Hill ex Maiden, de diferentes idades, visando a sua utilização na indústria moveleira. 2002. 160f. Tese (Doutorado em Ciências Florestais) Universidade Federal do Paraná, Curitiba, 2002.

TSOUMIS, G. Science and technology of wood: structure, properties and utilization. New York: Van Nastrnd Reinold, 1991. 494p. 